\title{
Experimental Determination of Effective Minority Carrier Lifetime in HgCdTe Photovoltaic Detectors Using Optical and Electrical Methods
}

\author{
Haoyang Cui, Jialin Wang, Chaoqun Wang, Can Liu, Kaiyun Pi, \\ Xiang Li, Yongpeng $\mathrm{Xu}$, and Zhong Tang
}

Shanghai University of Electric Power, Shanghai 200090, China

Correspondence should be addressed to Haoyang Cui; cuihy@shiep.edu.cn

Received 28 November 2014; Accepted 24 January 2015

Academic Editor: Wen Lei

Copyright (C) 2015 Haoyang Cui et al. This is an open access article distributed under the Creative Commons Attribution License, which permits unrestricted use, distribution, and reproduction in any medium, provided the original work is properly cited.

\begin{abstract}
This paper presents experiment measurements of minority carrier lifetime using three different methods including modified opencircuit voltage decay (PIOCVD) method, small parallel resistance (SPR) method, and pulse recovery technique (PRT) on pn junction photodiode of the $\mathrm{HgCdTe}$ photodetector array. The measurements are done at the temperature of operation near $77 \mathrm{~K}$. A saturation constant background light and a small resistance paralleled with the photodiode are used to minimize the influence of the effect of junction capacitance and resistance on the minority carrier lifetime extraction in the PIOCVD and SPR measurements, respectively. The minority carrier lifetime obtained using the two methods is distributed from 18 to $407 \mathrm{~ns}$ and from 0.7 to $110 \mathrm{~ns}$ for the different $\mathrm{Cd}$ compositions. The minority carrier lifetime extracted from the traditional PRT measurement is found in the range of 4 to $20 \mathrm{~ns}$ for $x=0.231-0.4186$. From the results, it can be concluded that the minority carrier lifetime becomes longer with the increase of $\mathrm{Cd}$ composition and the pixels dimensional area.
\end{abstract}

\section{Introduction}

Minority carrier lifetime is an important characteristic to evaluate the quality of photovoltaic material and the performance of the photoelectronic devices [1]. The basic theory of minority carrier recombination through recombination centers was put forth in 1952 [2]. Even though many techniques have been developed in order to determine the minority carrier lifetime for Si-based or Ge-based devices, the carrier lifetime for the HgCdTe photovoltaic infrared focal plane array (IRFPA) photodetector [3-6] is still a puzzling question, especially for the $p n$ junction devices. This is because the instability of $\mathrm{HgCdTe}$ material, in which the characteristics may change during the formation process of device, results in the differences between the actual and design parameters such as carrier concentration and the junction depth and so forth. These issues have many uncertainties effects on the minority carrier lifetime in the $p n$ junction device.

Previous studies results show that the lifetime obtained is widely distributed in the range of ns $\mu$ s [7] for different testing techniques, Cd composition, active junction area, and growth conditions. Since the investigation of the carrier lifetime is beneficial to understand the recombination mechanism, the measurements must be carried out on the actual devices to extract the minority carrier lifetime. Here, on the junction type HgCdTe IRFPA, we compare minority carrier lifetimes measured by PIOCVD method $[8,9]$, SPR method [10], and PRT [11] for a series of Cd compositions and the pixels dimensional area.

\section{Device Description and Experimental Setup}

The $\mathrm{Hg}_{1-x} \mathrm{Cd}_{x} \mathrm{Te}$ photovoltaic array detector was grown by MBE epitaxy on GaAs substrates. An ion implantation was fabricated on the $p$-type $\mathrm{HgCdTe}$ layer through $\mathrm{B}^{+}$ion implantation to form the $n^{+}$region. The acceptor and donor concentration were $N_{a} \approx 8 \times 10^{15} \mathrm{~cm}^{-3}$ and $N_{d} \approx 1 \times 10^{17}$ $\mathrm{cm}^{-3}$, respectively. The $n^{+}$heavy-doping area was $50 \times 50 \mu \mathrm{m}^{2}$ or $28 \times 28 \mu \mathrm{m}^{2}$. A ZnS film was evaporated on the surface 
as a passivation layer. The metal contacts were deposited on either side of the $n$-on- $p$ junctions in preparation for photovoltaic measurements. The sample was mounted in a liquid nitrogen-cooled Dewar for measurements and the temperature was close to $77 \mathrm{~K}$.

The transient photovoltage of the photodetector was stimulated by the incident laser pulse, which was generated by an optical parametric oscillator (OPO) and difference frequency generator (DFG) pumped with a picosecond Nd:YAG pulsed laser. The laser pulse duration was $30 \mathrm{ps}$ and the repetition rate was $10 \mathrm{~Hz}$. An Oriel THL (tungsten halogen lamp, Oriel 63355) was used as the bias light source to stimulate the steady-state photovoltage. The nanosecond electric pulses in the PRT measurement were generated by an Agilent 33250A. The sample under test was connected via BNC coaxial cable in series with a $50 \mathrm{Ohm}$ matched load resistance. An Agilent Infiniium 54832B oscilloscope was used to record the optical and electrical methods signals obtained.

\section{Lifetime Measurement Techniques and Results Discussions}

3.1. Photo-Induced Open-Circuit Voltage Decay Method. Theoretically, when the HgCdTe photodiode is excited by the pulsed laser, the photoresponse shows a rapid increase and slow decay process. As it has been analyzed in the previous report $[8,9]$, however, the decay curve profiles are dominated by the $R C$ discharge time constant and trap energy level capture effects on excess carrier's relaxation. In order to minimize the effects of the equivalent junction capacitor and the carrier traps in the $\mathrm{HgCdTe}$ photodiode, a constant background illumination has been introduced in the traditional OCVD method, which can be called photo-induced open-circuit voltage decay (PIOCVD) method.

The composition of $\mathrm{Hg}_{1-x} \mathrm{Cd}_{x} \mathrm{Te}$ in our experiments is $x=0.231\left(\lambda_{\mathrm{Eg}} \sim 8.6 \mu \mathrm{m}\right), x=0.305\left(\lambda_{\mathrm{Eg}} \sim 4.6 \mu \mathrm{m}\right), x=$ $0.343\left(\lambda_{\mathrm{Eg}} \sim 3.7 \mu \mathrm{m}\right)$, and $x=0.418\left(\lambda_{\mathrm{Eg}} \sim 2.9 \mu \mathrm{m}\right)$. The detectors were processed into $50 \times 50 \mu \mathrm{m}^{2}$ area of planar structures. By increasing the bias light intensity, the steadystate photovoltage will saturate the junction potential barrier of the photodiode. When the steady-state photovoltage does not increase with the rising of the background intensity, the photogenerated carriers recombination will dominate the decay time constant, which is related to the minority carrier lifetime. In agreement with this picture, photovoltage transient decay can be fitted by a one-order exponential function.

Figure 1 shows the situation that the DC photovoltage has been saturated. The photovoltage transient decay time constant can be determined from the best fit to the experiment, and consequently the minority carrier lifetime is obtained. The results show that the minority carrier lifetime is in the range of $18 \sim 407 \mathrm{~ns}$ for $x_{\mathrm{Cd}}=0.231 \sim 0.4186$ at $77 \mathrm{~K}$. With the $\mathrm{Cd}$ composition increasing, the minority carrier lifetimes show an increasing tendency. By comparing the lifetime values of different pixels in one-component array, it is found that there are some distinctions between the different pixels, which can be attributed to the nonuniformity of the $\mathrm{HgCdTe}$

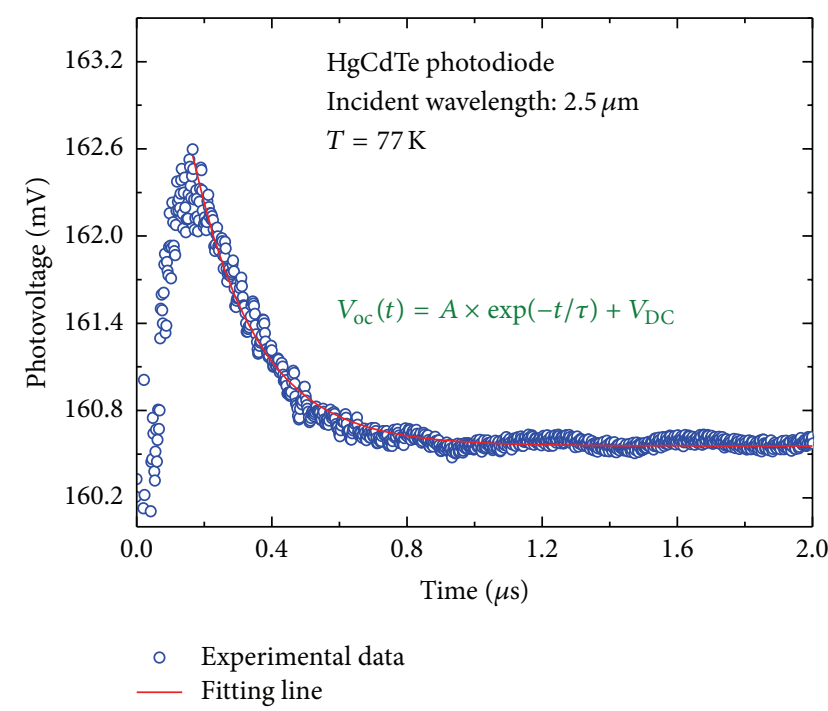

FIgure 1: Photovoltaic transient profile of the HgCdTe photodiode illuminated by the laser pulses and the saturate bias light intensity.

material or due to the fabrication process variations of the detector.

3.2. Small Parallel Resistance Method. In order to minimize the effects of the equivalent junction, we can parallel a small resistance in the load circuit of the photodiode in the transient photovoltage measurement, which is called the small parallel resistance (SPR) method [10]. In this situation, the load resistance combines with the junction series resistance to parallel with the junction shunt resistance. Although all these resistances contribute to the $R C$ discharge time constant and impact the photovoltage decay curve, the influence of the junction shunt resistance on the $R C$ constant can be minimized because the load resistance and the junction series resistance are much less than the junction shunt resistance. Thus, the delay effect of the $R C$ discharging process on the photovoltaic decay curve is weakened and the time constant will be dominated by the minority carrier recombination, consequently.

The dependence of bias light intensity on the photovoltage decay profile in SPR is very different from that in the PIOCVD measurement. Figure 2 is the photovoltage of the $\mathrm{HgCdTe}$ photodiode illuminated by laser pulses with different bias light intensity when a $50 \Omega$ resistance has been connected in the load circuit. Since the bias light illuminating nearly does not influence the transient photovoltage curves, $R C$ discharging can be excluded from the photovoltage decay process; therefore, the minority carrier lifetime can be correlated to the decay time constant. The area size measured in the experiment is $50 \times 50 \mu \mathrm{m}^{2}$ and $28 \times 28 \mu \mathrm{m}^{2}$. The oneorder exponential function can fit well with the experiment indicating that transient photovoltage decay is an exponential characteristic, which is shown in Figure 3. Thus, the photogenerated minority carrier lifetime can be extracted. Using this method, we measured the minority carrier lifetimes for different $\mathrm{Cd}$ components and different area dimensions of the 


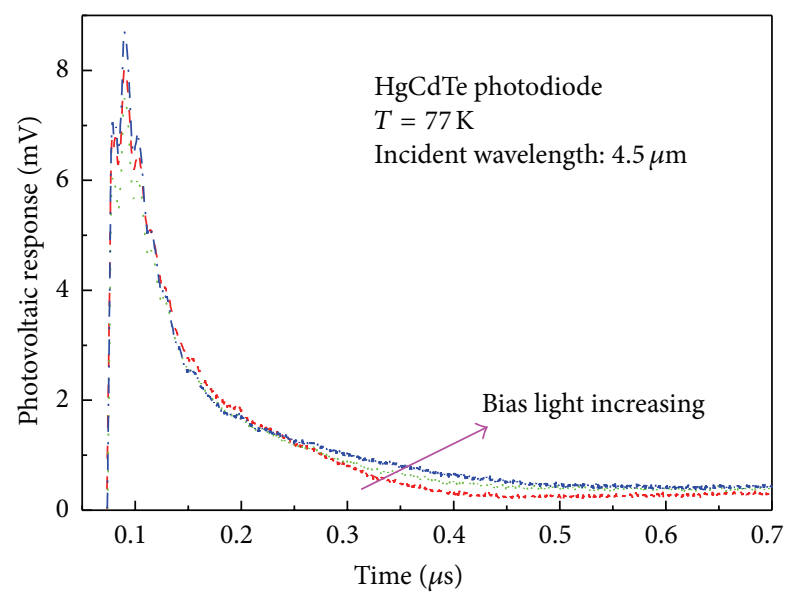

FIgURE 2: Photoresponse of the HgCdTe photovoltaic detector illuminated by laser pulses with different bias light intensity with a small resistance paralleled in the load circuit.

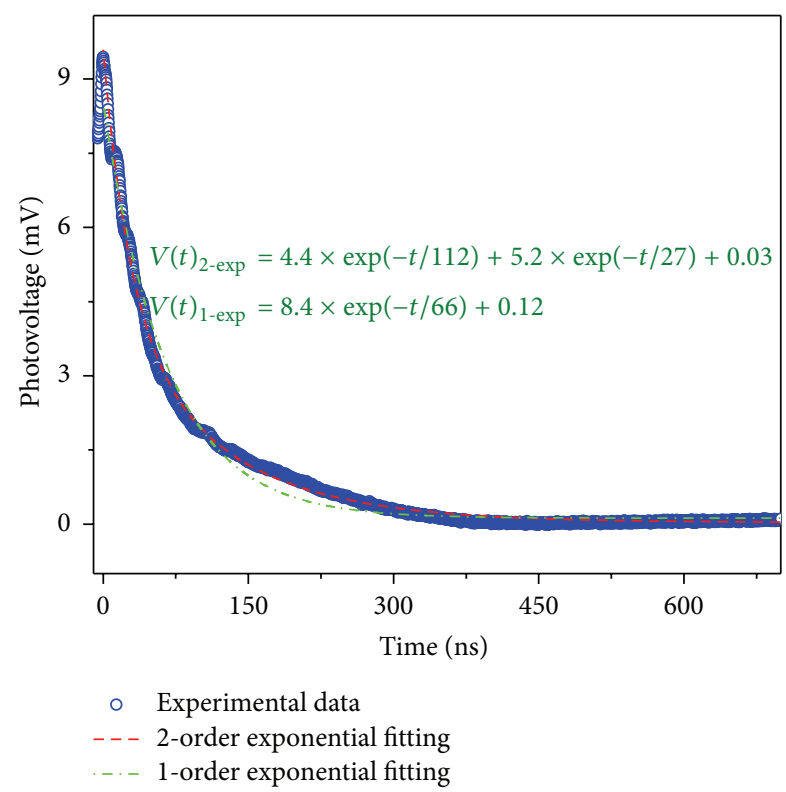

Figure 3: Photovoltaic profile of the detector illuminated by laser pulse.

$\mathrm{HgCdTe}$ photodiode. They show that the carrier lifetime is in the range of $0.7 \sim 110 \mathrm{~ns}$ at $77 \mathrm{~K}$. The lifetime becomes longer with the increase of $\mathrm{Cd}$ composition that is consistent with the PIOCVD measurement result. The results also show that the minority carrier lifetime decreases with decreasing area because of perimeter surface recombination effects, which coincides with previous works $[12,13]$.

3.3. Pulse Recovery Technique. The pulse recovery technique (PRT) [11] is a widely used method to determine the minority carrier lifetime in the pn junction diodes, which was developed in 1954. When the photodiode is rapidly switched from forward into reverse bias, the excess minority carriers which remained from forward bias injection must recombine

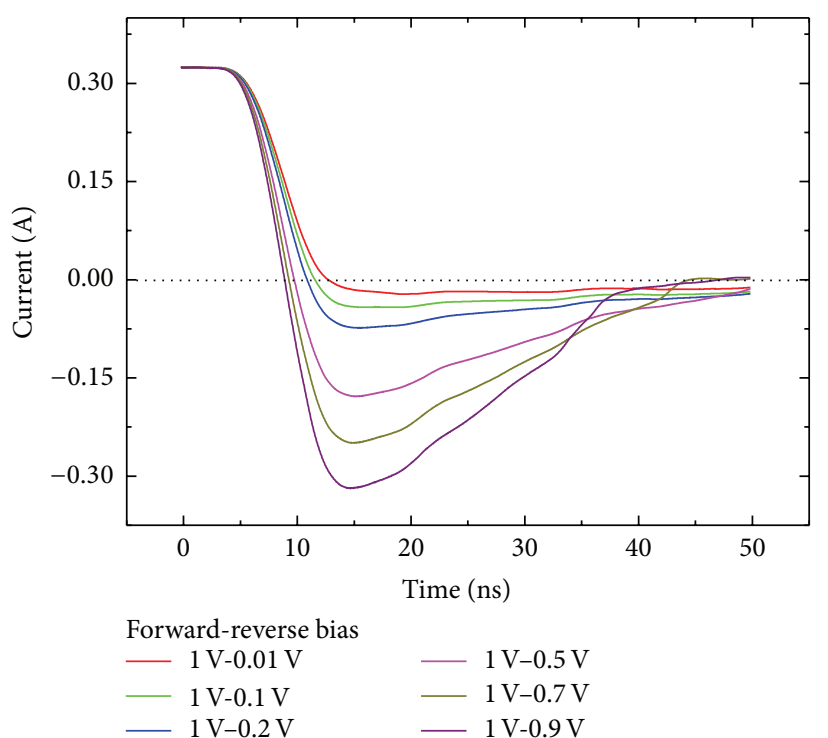

FIGURE 4: Reverse recovery current transient recorded on the diode as a function of varying initial reverse bias.

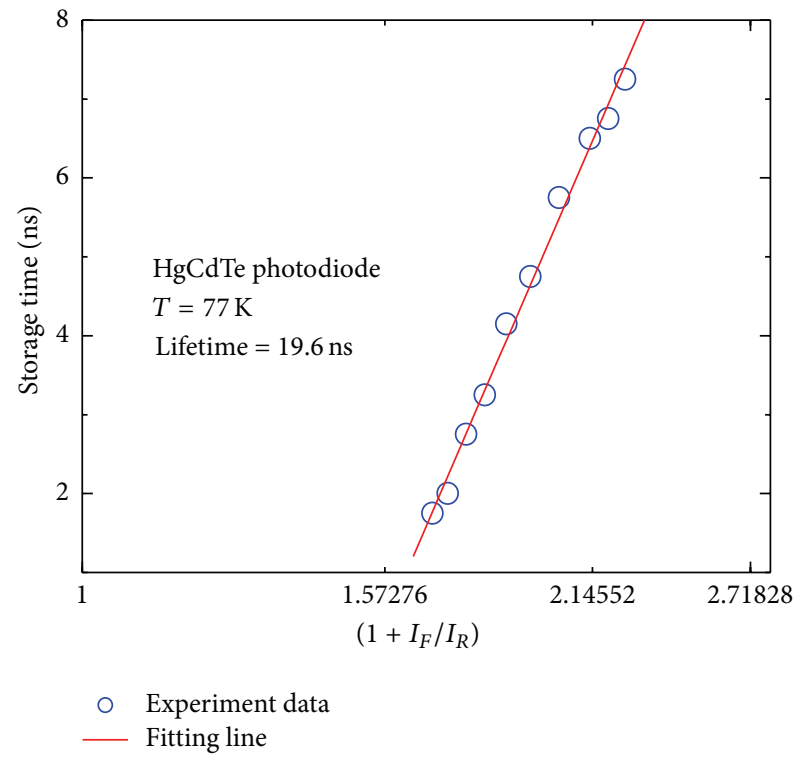

Figure 5: The storage time versus the current ratio $I_{F} / I_{R}$.

before current flow through the diode can drop to near zero [14]. There are two different decay phases which can be distinguished in the reverse current signals: one is the constant recovery current component and the other one is the reverse current dropping to near zero subsequently. Figure 4 shows the reverse recovery current transient recorded on the device as a function of varying initial reverse bias. The average minority carrier lifetime $\tau$ can be calculated from $t_{s}$ and the ratio of forward current to reverse current, which is shown in Figure 5.

The active area of the devices used in this experiment is $50 \times 50 \mu \mathrm{m}^{2}$ and $28 \times 28 \mu \mathrm{m}^{2}$. The lifetime distributes in the range of 4 to $30 \mathrm{~ns}$ for $x_{\mathrm{Cd}}=0.231 \sim 0.419$ at $77 \mathrm{~K}$. The 


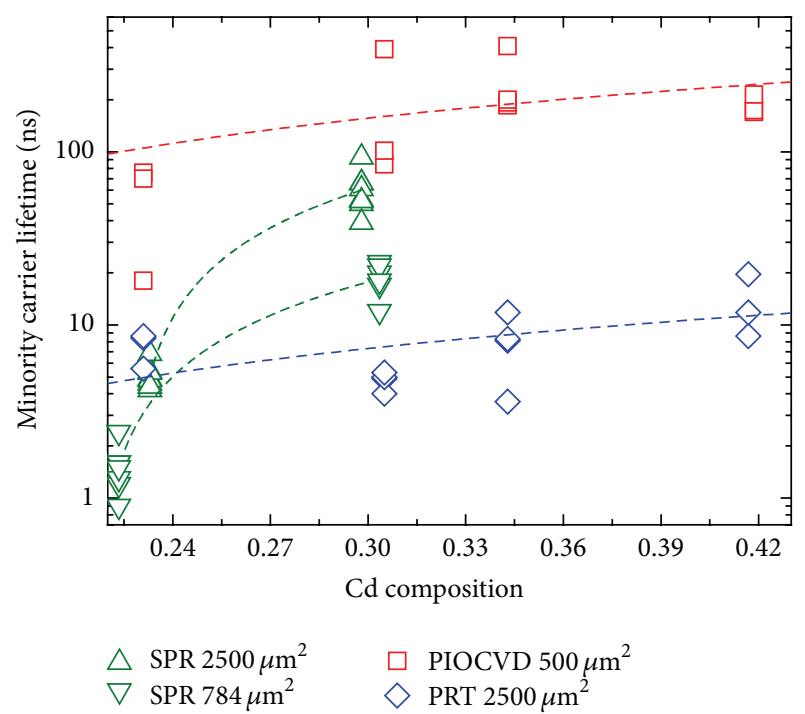

FIGURE 6: The variation of the extracted lifetime values with $\mathrm{Cd}$ composition using three experimental methods.

results also show that the value of the lifetime becomes longer with the increase of $\mathrm{Cd}$ composition. This is also consistent with the PIOCVD and SPR measurement results.

3.4. Results Discussions. From the above discussion, we measured the $\mathrm{HgCdTe}$ photodiode with different $\mathrm{Cd}$ compositions and the pixels dimensional area using the three different techniques including PIOCVD, SPR, and PRT. The minority carrier lifetime extracted from these methods is summarized and shown in Figure 6. It is clear that no matter which kind of experiment methods is adopted the minority carrier lifetime values obtained from these methods show the same increasing trend with the increase of $\mathrm{Cd}$ compositions. The values of the minority carrier lifetime show the increase with the increase of $\mathrm{Cd}$ composition. On the other hand, the minority carrier lifetimes extracted from the three methods are unequal. For example, the carrier lifetime obtained from PIOCVD is sometimes bigger than the ones from PRT. This is because the $R C$ effect cannot be avoided in a $p n$ junction photodiode no matter in what bias-light conditions in PIOCVD measurement. Since the $R C$ discharging process delays the photovoltage decay cure, the minority carrier lifetime is bigger than the real values. In addition, the entire region of the device is in a high-injection condition, and the minority carrier lifetimes are larger than low injection [1], consequently. For the PRT measurements, recombination theory dictates that the minority carrier lifetime will be affected seriously by the surface recombination. Particularly, for the thin base region photovoltaic device, its dominance effect is even more pronounced. The effective minority lifetime profile can be given by including both surface recombination and diffusion of carriers:

$$
\tau_{\text {eff }}=\frac{d}{S}+\frac{d^{2}}{\pi^{2} D}
$$

where $d$ is the simple thickness perpendicular to the surface plane, $S$ is the surface recombination velocity, and $D$ is the diffusion coefficient. This equation describes the effective carrier lifetime as a function of depth due to the surface recombination and diffusion of carriers within the sample to the surface. In this reason, the measured value is smaller than the base region value. Another correlate point to be cleared up is the influence of pixels dimensional area on the minority carrier lifetime measurement. Form Figure 6, one can see that the minority carrier lifetime of $\mathrm{HgCdTe}$ photodiode with the pixels dimensional area of $2500 \mu \mathrm{m}^{2}$ is larger than that with the pixles dimension area of $784 \mu \mathrm{m}^{2}$ in the SPR measurements. Because the HgCdTe IRFPA was fabricated to the planar junction structure, the active area is related to the junction area. Large decrease in the lifetime with decreasing device size suggests that surface recombination plays an important role in limiting effective device minority carrier lifetime which may be interpreted as the effect of specific surface area on the minority carrier lifetime [15]. The smaller the pixel dimension area is, the larger the specific surface area is, and the greater the effect of surface recombination is, consequently.

\section{Conclusion}

In summary, the work described in this paper presented the two kinds of minority carrier lifetime optical measurement methods including the PIOCVD method and the SPR method and the electrical method based on PRT. In the optical measurement method, a constant bias light illumination and a small resistance parallel in the load circuit were used to minimize the junction $R C$ discharging process, respectively. The measurement results of the three methods show that the values of the minority carrier lifetime will increase with the increase of Cd compositions. It also shows that the lifetime of PIOCVD is larger than that of PRT, which can be attributed to the $R C$ discharging effect in PIOCVD and strong surface recombination effect in PRT. The experiment methods and the conclusion of this paper will have significance for the $\mathrm{HgCdTe}$ device design and optimization.

\section{Conflict of Interests}

The authors declare that they have no conflict of interests related to this work.

\section{Acknowledgment}

The project is supported by the National Natural Science Foundation of China (61107081).

\section{References}

[1] S. Johnston, K. Zaunbrecher, R. Ahrenkiel, D. Kuciauskas, D. Albin, and W. Metzger, "Simultaneous measurement of minority-carrier lifetime in single-crystal CdTe using three transient decay techniques," IEEE Journal of Photovoltaics, vol. 4, no. 5, pp. 1295-1300, 2014. 
[2] D. K. Schröder, "Carrier lifetimes in silicon," IEEE Transactions on Electron Devices, vol. 44, no. 1, pp. 160-170, 1997.

[3] W. C. Qiu and W. D. Hu, "Laser beam induced current microscopy and photocurrent mapping for junction characterization of infrared photodetectors," Science China Physics, Mechanics \& Astronomy, vol. 58, no. 2, pp. 1-13, 2015.

[4] W.-D. Hu, X.-S. Chen, Z.-H. Ye et al., "Dependence of ionimplant-induced LBIC novel characteristic on excitation intensity for long-wavelength HgCdTe-based photovoltaic infrared detector pixel arrays," IEEE Journal on Selected Topics in Quantum Electronics, vol. 19, no. 5, Article ID 4100107, 2013.

[5] W. Hu, Z. Ye, L. Liao et al., "A 128×128 long-wavelength/midwavelength two-color HgCdTe infrared focal plane array detector with ultra-low spectral crosstalk," Optics Letters, vol. 39, pp. 5130-5133, 2014.

[6] J. Liang, W. Hu, Z. Ye et al., "Improved performance of HgCdTe infrared detector focal plane arrays by modulating light field based on photonic crystal structure," Journal of Applied Physics, vol. 115, no. 18, Article ID 184504, 2014.

[7] D. Y. Tang and Z. Y. Mi, Introduction to Optoelectronic Devices, Shanghai Science and Technology Literature Press, 1989.

[8] H. Cui, Z. Li, Z. Ye, X. Hu, and W. Lu, "Methods for determining minority carrier lifetime in HgCdTe photovoltaic detectors," in Proceedings of the 34th International Conference on Infrared, Millimeter, and Terahertz Waves (IRMMW-THz'09), September 2009.

[9] H. Cui, N. Tang, and Z. Tang, "Experimental determination of minority carrier lifetime and recombination mechanisms in MCT photovoltaic detectors," in Proceedings of the 12th International Conference on Numerical Simulation of Optoelectronic Devices (NUSOD '12), pp. 39-40, August 2012.

[10] H. Cui, C. Wang, J. Wang et al., "Measurement of minority carrier lifetime in infrared photovoltaic detectors using parallel circuit method," Optical and Quantum Electronics, 2014.

[11] V. K. Khanna, "Physical understanding and technological control of carrier lifetimes in semiconductor materials and devices: a critique of conceptual development, state of the art and applications," Progress in Quantum Electronics, vol. 29, no. 2, pp. 59-163, 2005.

[12] P. J. Drummond, D. Bhatia, and J. Ruzyllo, "Measurement of effective carrier lifetime at the semiconductor-dielectric interface by Photoconductive Decay (PCD) Method," Solid-State Electronics, vol. 81, pp. 130-134, 2013.

[13] H. Jeong, H. Song, Y. Pak et al., "Enhanced light absorption of silicon nanotube arrays for organic/inorganic hybrid solar cells," Advanced Materials, vol. 26, no. 21, pp. 3445-3450, 2014.

[14] D. L. Polla, S. P. Tobin, M. B. Reine, and A. K. Sood, "Experimental determination of minority-carrier lifetime and recombination mechanisms in p-type $\mathrm{Hg}_{1-x} \mathrm{Cd}_{x}$ Te," Journal of Applied Physics, vol. 52, no. 8, pp. 5182-5194, 1981.

[15] P. G. Neudeck, "Perimeter governed minority carrier lifetimes in $4 \mathrm{H}-\mathrm{SiC} \mathrm{p}^{+} \mathrm{n}$ diodes measured by reverse recovery switching transient analysis," Journal of Electronic Materials, vol. 27, no. 4, pp. 317-323, 1998. 

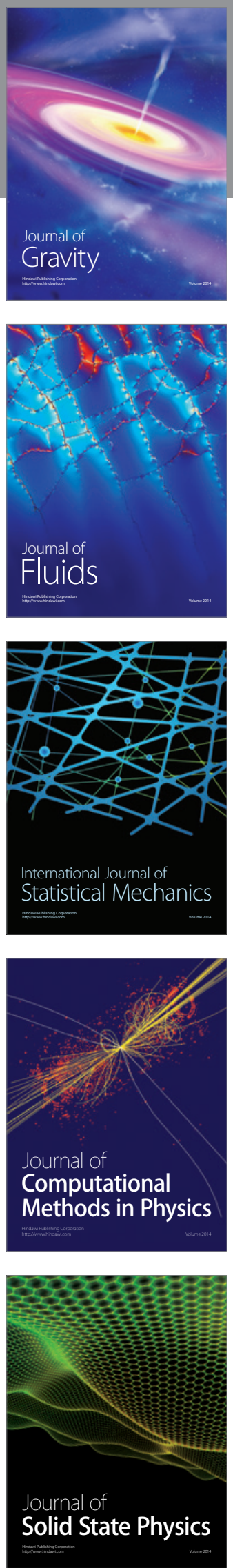

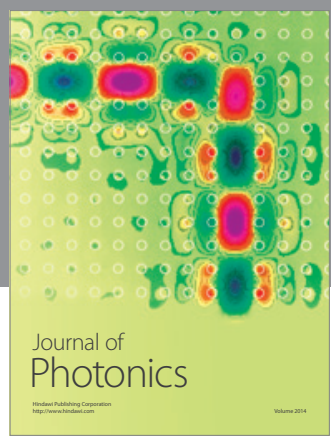

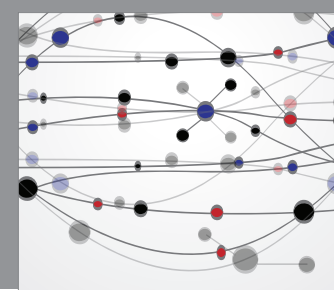

The Scientific World Journal

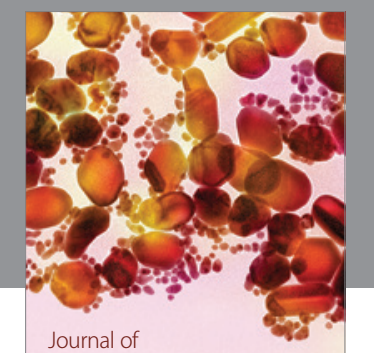

Soft Matter
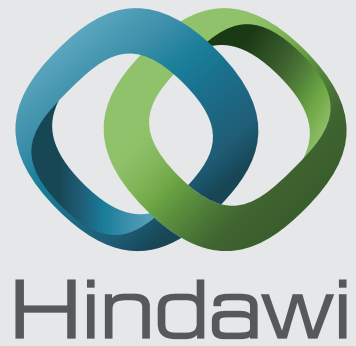

Submit your manuscripts at

http://www.hindawi.com
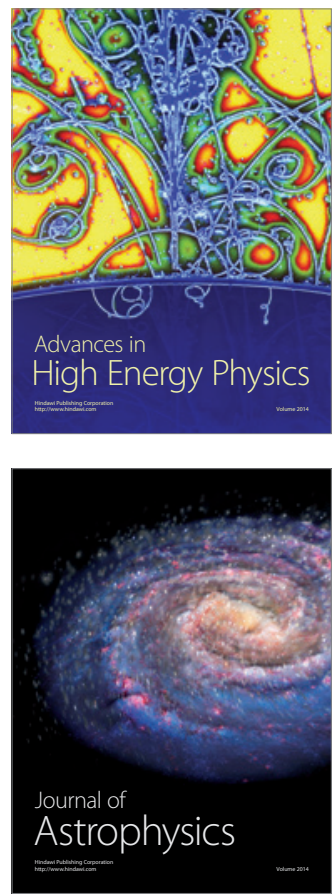
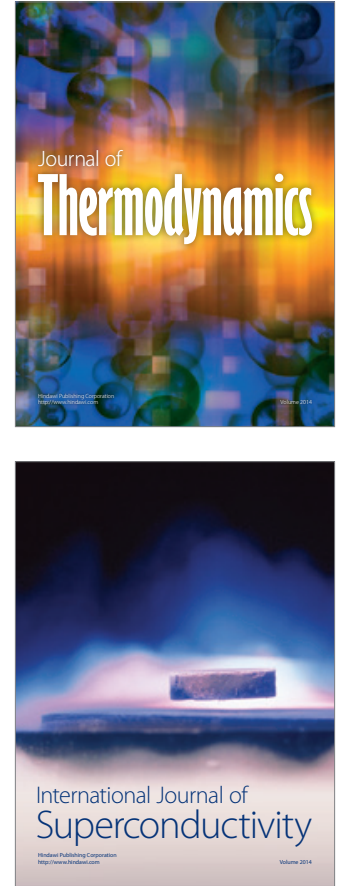
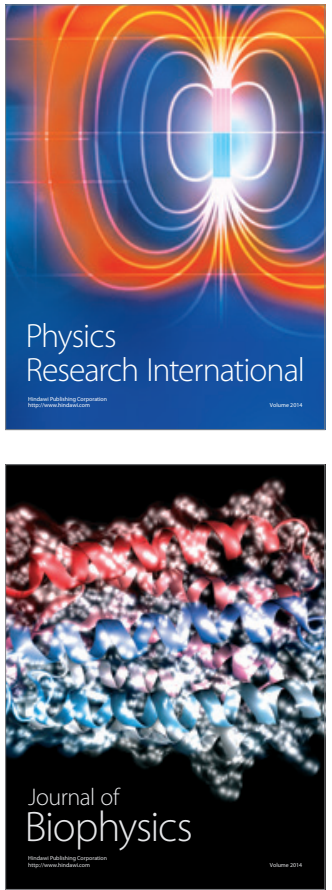
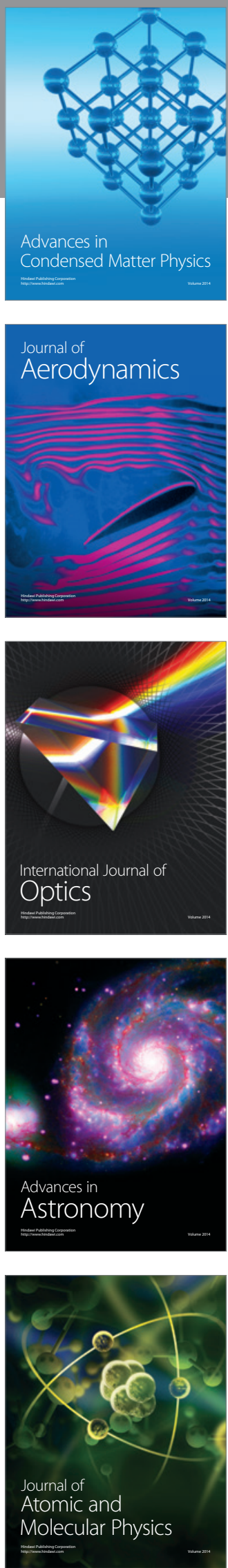